\title{
Actuación de enfermería ante un paciente con calcifilaxis
}

\section{Dolores Piña Simón* - Alicia González Horna* - Ma Carmen Gálvez Serrano* - Belén Marco García* - Esther Rubio González** -}

*Diplomado Universitario en Enfermería - ** Nefrólogo

Centro de hemodiálisis "Los Llanos". Fundación Renal Iñigo Álvarez de Toledo (FRIAT).Móstoles. Madrid

\section{Introduccion}

La arteriolopatía urémica calcificante, también conocida como calcifilaxis, produce calcificación con proliferación de la íntima, fibrosis y trombosis. Clínicamente se caracteriza por isquemia y necrosis de la piel, tejido subcutáneo y a veces tejidos adyacentes, pudiendo afectar también a otros parénquimas como corazón, articulaciones, pulmones, páncreas, intestino y ojo'; afecta del $1 \%$ al $4 \%$ de la población en diálisis. Fundamentalmente, se desarrolla en pacientes con insuficiencia renal en estadío 5.

A pesar de los avances en las técnicas dialíticas, el pronóstico es pésimo, con morbilidad asociada a infecciones y mortalidad del $80 \%$ de los casos en pocos meses por compromiso séptico y disfunción de los órganos afectados, no existiendo en la actualidad un tratamiento eficaz ${ }^{2}$.

Aunque las alteraciones propias del estado urémico y del metabolismo $\mathrm{Ca} / \mathrm{P}$ son importantes, la etiopatogenia es compleja siendo los mecanismos desencadenantes poco conocidos. Suele afectar con más frecuencia a pacientes mayores, sexo femenino, raza blanca, diabéticos y en casos aislados han sido descritos en hiperparatiroidismo primario, Crohn, SIDA y cirrosis 3 .

Correspondencia:

Dolores Piña Simón

Centro de Hemodiálisis LOS LLANOS. FRIAT

C/ Río Segura ${ }^{\circ} 5$

28935, Móstoles (Madrid)

Ilanos@friat.es
Como factores de riesgo de la calcifilaxis descritos en la literatura son ${ }^{1}$ :

- Producto Ca/P aumentado

- Uso de análogos de vitamina D y warfarina

- Deficiencia de vitamina K

- Deficiencia de proteína C y S

- Obesidad

- Desnutrición proteica

- Dicumarínicos

El síntoma más llamativo es el dolor. Además de la aparición de lesiones ulceradas de borde violáceo que evolucionan a úlceras necróticas. Se confirman posteriormente en algunos casos con estudios radiológicos e histológicos. Generalmente la calcifilaxis se presenta con púrpura reticuliforme aunque otros cuadros clínicos pueden ser similares histológicamente como ${ }^{4}$ :

- Vasculitis sistémicas

- Crioglobulinemia

- Ateroesclerosis periférica

- Vasculopatías trombóticas

- Síndrome anticoagulante lúdico

- Déficit proteína C y S

- Necrosis por dicumarínicos

- Embolismo del colesterol.

\section{Historia clinica}

Mujer de 70 años de edad con antecedentes patológicos de: 
- IRC secundaria a nefroangioesclerosis en tratamiento renal sustitutivo con hemodiálisis desde noviembre de 2002

- HTA de larga evolución

- Fibrilación auricular con respuesta ventricular rápida y elevación de enzimas cardiacas. Sometida a anticoagulación

- Síndrome coronario agudo sin lesiones coronarias significativas

- Hiperparatiroidismo secundario muy severo que requiere paratiroidectomía en febrero 2005

- Desnutrición calórico-proteica

- Hipotiroidismo en tratamiento hormonal sustitutivo

- Hipercolesterolemia

- Se dializaba 3 días/semana durante 4 horas, con dializador de alta permeabilidad y baño de calcio 3; $\mathrm{K} 1,5$. Fístula arteriovenosa radio-cefálica derecha, con buena tolerancia hemodinámica a las sesiones.

En enero 2008 presenta placa dolorosa no ulcerada en zona externa de gemelo derecho con reacción inflamatoria periplaca violácea. Valorada por cirugía vascular descartan patología arterio-venosa. Ante las características de la lesión y la evolución de la misma se diagnostica de calcifilaxis. Tras consulta con el servicio de dermatología decide no realizar biopsia por el alto riesgo de empeoramiento de la lesión. Al diagnóstico la paciente presentaba buen control del producto $\mathrm{Ca} / \mathrm{P}$ y con $\mathrm{PTH}$ de $10 \mathrm{pg} / \mathrm{ml}$ y fosfatasa alcalina de $123 \mathrm{U} / \mathrm{L}$.

\section{Plan de actuacion ante calcifilaxis}

- Se pautan HD diarias de 2,30 horas de duración y baño bajo en calcio $(2,5 \mathrm{mEq} / \mathrm{l})$.

- La paciente no recibía tratamiento con vitamina D ni quelantes cálcicos.

- Se suspende dicumarínico y se anticoagula con heparina de bajo peso molecular (bemiparina sódica) en las sesiones de HD.

- Se inicia tratamiento con $50 \mathrm{ml}$ de tiosulfato sódico al $25 \%$ iv en la última media hora de sesión, con buena tolerancia hemodinámica, pauta que se mantiene durante un año.

- Control del dolor con seguimiento por parte de la unidad del dolor: se inicia analgesia con paracetamol y metamizol, requiriendo posteriormente parches de fentanilo.

- Iniciamos un protocolo de cura de las lesiones que incluye :

- Medición del tamaño de las úlceras.

- No desbridar por un alto riesgo de infecciones y sangrado.

- Valorar signos de infección por si precisara antibióticos.

- Realización de las curas cada 72 horas.

\section{Evolucion de las ulceras}

Enero 2008: aparición de un nódulo de placa dolorosa y no ulcerada de 3-4 semanas de evolución en zona posterior del gemelo derecho, con reacción inflamatoria periplaca que ha ido a más en los últimos días. Pulso pedio derecho presente aunque más débil en el lado izquierdo, la temperatura de ambas piernas conservada (figura 1).

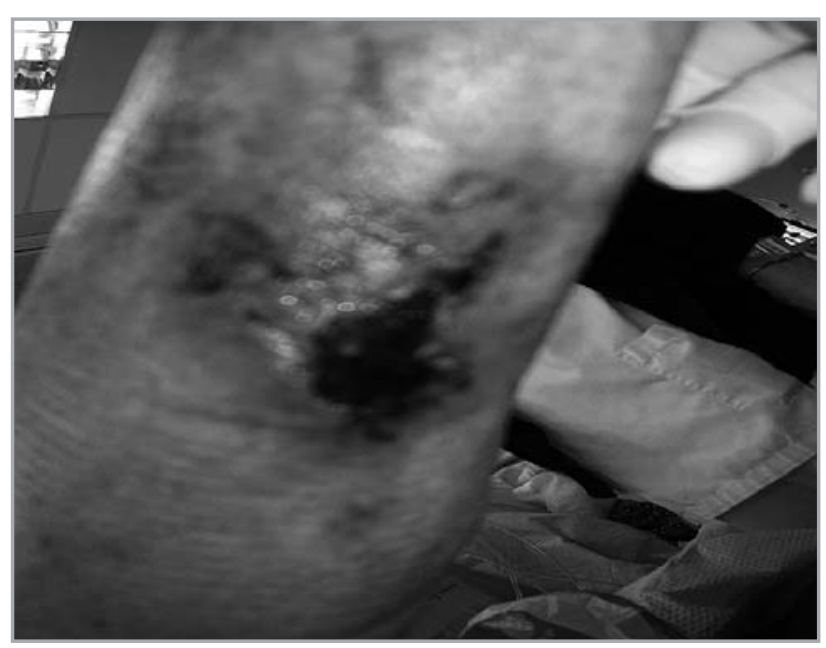

Figura 1: Úlcera necrótica de 4 semanas de evolución en gemelo derecho

Se realiza estudio de hipercoagulabilidad completo para descartar déficit de proteína C y S, antifosfolípidos. El fósforo es normal. El estudio inmunológico para 
descartar vasculitis es negativo. Se inicia tratamiento con tiosulfato sódico y se pautan parches Durogesic ${ }^{\circledR}$ (fentanilo) cada 72 horas para control del dolor.

Para la cura de la herida se inician medidas higiénicas posturales y curas locales y se realizan las curas cada 72 horas con suero fisiológico para limpieza de las úlceras, posteriormente con Prontosan ${ }^{\circledR}$ (producto para la limpieza, humectación y descontaminación de heridas cutáneas crónicas, contaminadas), colocando después el apósito hidrocoloide Varihesive ${ }^{\circledR}$ ). Se hace vendaje con venda de algodón Velband ${ }^{\circledR}$ y venda elástica de crepé hipoalérgica Baxcrepe Plus ${ }^{\circledR}$, para evitar roces y golpes en la zona. En este caso no precisa administración de antibióticos ante la ausencia de signos de infección.

Febrero 2008: la úlcera tiene mejor aspecto que días anteriores. Aparecen nuevas úlceras necróticas en la cara interna y externa de la pierna derecha. Se limpian las úlceras con suero fisiológico y Prontosan ${ }^{\circledR}$. Una vez se desprenden las placas necróticas, se cubren las úlceras con el apósito de hydrofiber con plata Aquacel plata ${ }^{\circledR}$ humedecido con suero fisiológico y se tapan con Varihesive ${ }^{\circledR}$, protegiendo la zona de la herida con venda de algodón Velband ${ }^{\circledR}$ y vendas elásticas de crepé hipoalérgica Baxcrepe Plus ${ }^{\circledR}$ ).

A finales de mes la evolución de las úlceras es favorable con tejido de granulación abundante y placa necrótica a punto de caerse (figura 2).

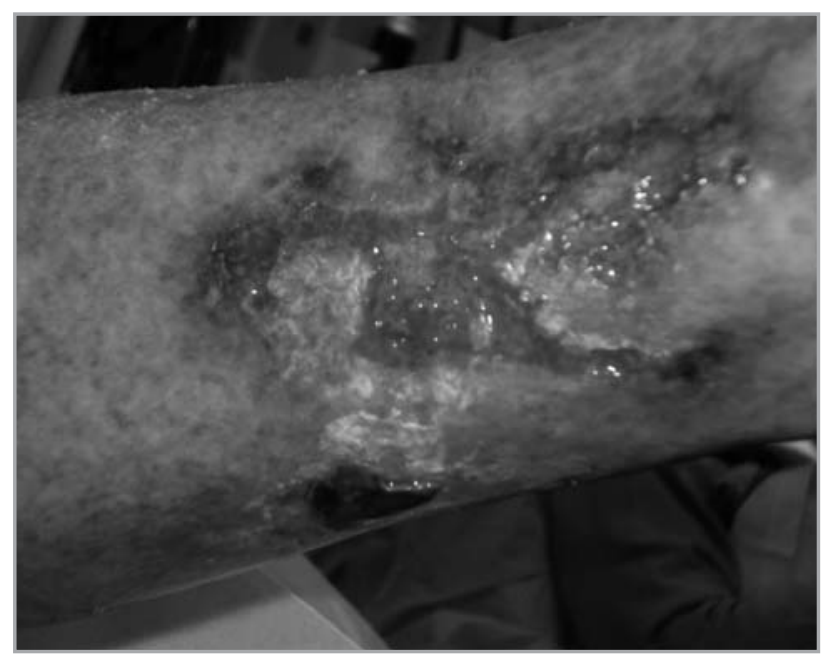

Figura 2: Tejido granuloso abundante y placa necrótica a punto de caerse
Marzo 2008: a lo largo del mes se desprende los restos de placa necrótica en la úlcera posterior de la pierna derecha con buena evolución del resto de las úlceras. Se continúa con la misma pauta de curas. A finales de este mes desaparece el dolor por lo que se suspenden parches Durogesic ${ }^{\circledR}$.

Abril 2008: ante la ausencia de placa necrótica y con tejido de granulación abundante en todas las úlceras, modificamos pauta de cura, prescindiendo del apósito hidrocoloide, en cuanto al Aquacel plata ${ }^{\circledR}$ lo pusimos sin humedecer siendo mal tolerado por la paciente con aparición de nuevo del dolor, por lo que volvemos a impregnar el mismo con suero fisiológico cubriéndolas con venda de algodón y vendas elásticas de crepé hipoalérgica.

Mayo 2008: las úlceras prácticamente han desaparecido, con abundante tejido de granulación y piel nueva revistiéndolas, por lo que se modifica pauta de curas limpiándolas con suero fisiológico y Prontosan ${ }^{\circledR}$ y cubriéndolas con apósitos de Tulgrasum cicatrizante ${ }^{\circledR}$. Continuamos con esta pauta hasta cicatrización total de las úlceras (figura 3). La evolución de la analítica desde el diagnostico hasta seis meses de evolución se muestra en la tabla 1 .

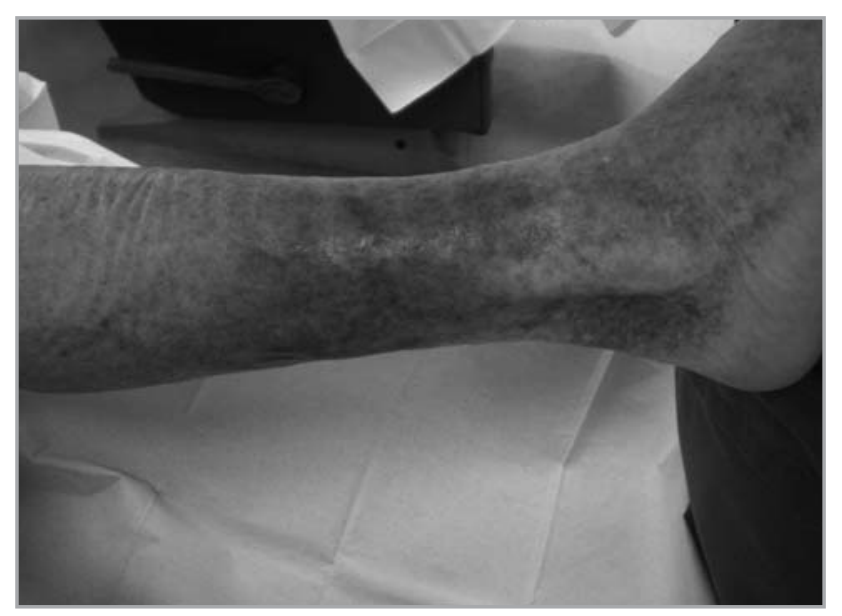

Figura 3: Cicatrización total de las ulceras

En el caso clínico que describimos la paciente presenta como factores de riesgo para la calcifilaxis estar en tratamiento con dicumarínicos desde el año 2004; los niveles de PTH eran bajos $( \pm 4.9)$ ya que estaba 


\begin{tabular}{|l|c|c|c|c|c|c|c|c|}
\hline & Media & Desv Est & ene-08 & feb-08 & mar-08 & abr-08 & may-08 \\
\hline Calcio corregido (mg/dl) & 9,2 & 0,4 & 8,90 & 8,90 & 9,70 & 8,80 & 9,00 \\
\hline Fósforo (P) (mg/dl) & 5,3 & 1,4 & 6,00 & 3,70 & 4,70 & 7,00 & 6,10 \\
\hline Producto CaxP & 48,1 & 12,6 & 53,40 & 32,93 & 45,59 & 61,60 & 54,90 \\
\hline PTH-i (pg/ml) & 4,3 & 4,9 & & 10,00 & 2,00 & 58,56 \\
\hline Fosfatasas Alcalinas (U/L) & 115,5 & 14,4 & 123,00 & & 121,00 & 1,00 \\
\hline Albúmina (g/dl) & 3,7 & 0,2 & 3,80 & 3,60 & 3,50 & 3,80 & 3,90 \\
\hline
\end{tabular}

Tabla 1. Evolución de la analítica

paratiroidectomizada desde el año 2005, producto $\mathrm{Ca} / \mathrm{P}$ eran normales $( \pm 12.6)$ siendo controlado con Sevelamer.

Una vez aparecidas las úlceras necróticas en la paciente y tras ser diagnosticada de calcifilaxis y ante las medidas terapéuticas adoptadas observamos que la respuesta de la paciente a la analgesia habitual con paracetamol y metamizol fue pobre y sólo conseguimos disminuir el dolor con parches de fentanilo. No aparecieron infecciones por lo que no fue necesaria la administración de antibióticos.

La pauta dialítica contempló el calcio en baño de diálisis a $2.5 \mathrm{mEq} / \mathrm{l}$ y aumentar las sesiones de diálisis a cinco semanales de 2,30 horas de duración; se administró tiosulfato sódico al $25 \%$ iv en la última media hora de sesión. El protocolo de curas de la herida se demostró efectivo.

El tratamiento de la calcifilaxis no está bien establecido y aunque se han descrito múltiples intervenciones con potencial terapéutico, ninguna de ellas es consistente. En bibliografía se han descrito tratamientos para la calcifilaxis como el uso de la cámara hiperbárica6 0 la aplicación de larvas estériles de Lucilia sericata $\left(\operatorname{LarvE}^{\circledR}\right)^{7}$. En cualquier caso el tratamiento tópico de las lesiones cutáneas continúa siendo uno de los pilares fundamentales.

Aunque no podemos demostrar que la pauta de cura descrita en nuestro caso clínico haya sido lo único que ha llevado a la mejoría progresiva de las úlceras por la coexistencia de otros tratamientos médicos y dialíticos coadyuvantes, sugiere la evolución favorable de la calcifilaxis. Actualmente estamos tratando otro caso de calcifilaxis, siguiendo el mismo protocolo de tratamiento con excelentes resultados.

\section{Bibliografía}

1. Munar MA, Alarcón A, Bernabéu R, Morey A, Gascó J, Losada P, Antón E, Marco J. A propósito de un caso de lesiones cutáneas y calcifilaxis. Nefrología 2001; 21: 501-504.

2. Robinson-Boston et al. Cutaneous manifestations of end-stage renal disease, J Am Acad Dermatol 2000; 43:975-986.

3. Esary et al. Cutaneous Calciphylaxis. Am J Clin Pathol 2000; 113; 280-287.

4. Llach. Calcific Uremi Arteriolopathy (Calciphylaxis). Am J Kidney Dis 1998:514-518.

5. González A, Piña D, Gálvez MC, Gago MC, Sánchez J $\mathrm{R}$, Martínez $\mathrm{S}$ et al. Manifestaciones dérmicas en hemodiálisis. Cuidados de enfermería ¿Nuevo enfoque? Rev Soc Esp Enfer Nefrol 2005; 8 (4): 75-78.

6. Vila Vidal D. Cámara hiperbárica. Planificación estandarizada de cuidados de enfermería. Revista de enfermería Rol 2002; 25(6):21-30.

7. Picazo M, Bover J, de la Fuente J, Sanz R, Cuxart M, Matas M. Larvas estériles como coadyuvantes al tratamiento local en una paciente con calcifilaxis proximal. Nefrología 2005; 25 (5): 559-562. 